\title{
AUTOMATIC SHIP BERTHING USING PARALLEL NEURAL CONTROLLER
}

\author{
Namkyun Im*, Kazuhiko Hasegawa** \\ **Osaka University, Osaka, Japan \\ Email : hase@naoe.eng.osaka-u.ac.jp \\ *Undergraduate student of Osaka Univ., Osaka, Japan \\ Email:im@ naoe.eng.osaka-u.ac.jp
}

\begin{abstract}
In this paper a parallel ANN(artificial neural networks) for the automatic berthing will be discussed. This controller has a separated hidden layer each control an engine and a rudder respectively. Using this controller simulations were carried out where the initial conditions such as ship's positions and heading angle are different from teaching data. Finally comparison of separated hidden layer and united hidden layer will be described. Copyright $(2001$ IFAC
\end{abstract}

Key words: parallel neural networks, automatic controllers, artificial intelligence,

expert systems, fuzzy control.

\section{INTRODUCTION}

Automatic berthing control is one of unresolved problems in ship control. Many methods and theories were adopted to achieve the goal. A typical example is feedback control, which has been used as a controller in some research. (K. Kose et al.,1986). Even if conventional feedback controller is great tool, ship's berthing is so complicated that a lot of limitations still are found. Therefore many studies (Koyama.1987, Yamato.1990\&1992, Hasegawa.1993, Itoh 1998) suggest other controllers such as fuzzy theory, neural network, and expert system. A neural network controller is one of them. As it is known, a neural network has good ability to recognize the pattern and then produces similar output with the pattern. The feature was used to control a ship in berthing problem. Yamato (H. Yamato,1990) suggested automatic berthing by a neural network and produced excellent results, but he soon changed his research field to others such as expert system (H. Yamato,1992). So deep study for neural networks controller was not conducted. Hasegawa (K. Hasegawa,1993) took over the study. Excellent results were produced even if many things should be overcome: general simulations have same initial value with teaching data and oscillation phenomenon occurred in controller. In other hands, when you look to the existing research that is done for the automatic berthing, it is found that main 


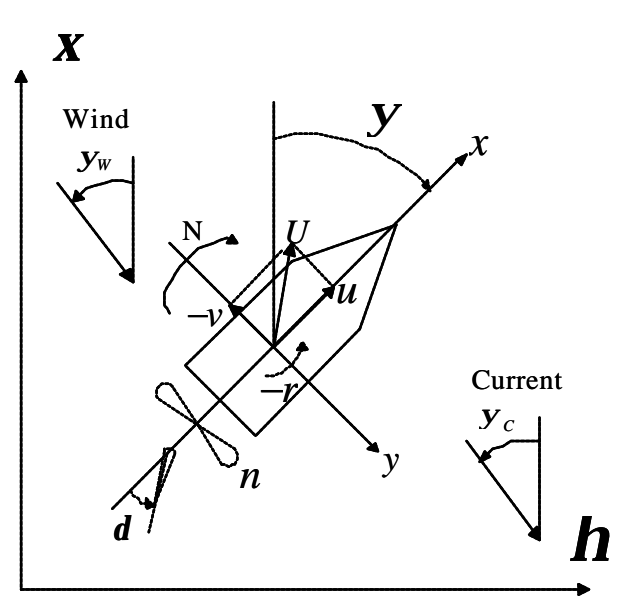

Fig. 1 Coordinate system for ship dynamics

concept is based on a centralized controller. In other words, output units just come from one command line system. Some studies (Itoh, 1998) mentioned that the concept of centralized control might make the problem more difficult. This paper is based on the same idea. Parallel order system in the ANN is suggested. Two feed forward networks are adopted to compose parallel ANN.

\section{ARCHITECTURE OF ANN}

\subsection{Model ship}

A 260,000 tons of tanker is adopted for this paper, of which dynamics and details are well explained in other research (K. Kose,1986). Particulars of the ship are presented in table 1 and dynamics coordinate is given in Fig. 1.

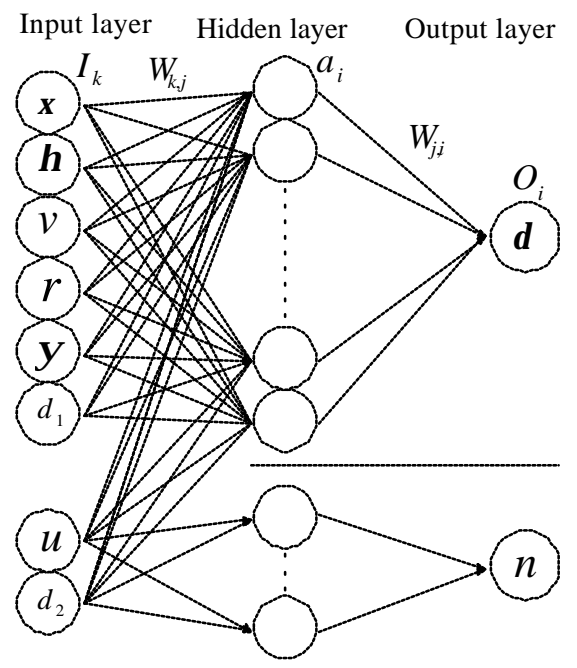

Fig. 2 Neural network with parallel hidden layer
Table 1 Particulars of ship

\begin{tabular}{lll}
\hline Hull & Ship type & Tanker \\
& Length & $304(\mathrm{M})$ \\
& Beam & $52.5(\mathrm{M})$ \\
& Draft & $17.4(\mathrm{M})$ \\
& $\mathrm{Cb}$ & 0.827 \\
\hline \multirow{2}{*}{ Propeller } & Rudder Height & $12.94(\mathrm{M})$ \\
& Propeller Diameter & $8.5(\mathrm{M})$ \\
& Propeller Pitch & $5.16(\mathrm{M})$ \\
& Rudder area & $98\left(\mathrm{M}^{2}\right)$ \\
& Pitch ration & 1.709 \\
\hline
\end{tabular}

\section{$2.2 A N N$}

A typical three-layer network is used. The main future is the separated structure of hidden layer as shown in Fig. 2. As mentioned in introduction, a parallel control is adopted in neural network. A conventional neural network in ship berthing problem just has one of hidden layer. But the neural network in this paper has separated hidden layer that controls engine and rudder respectively. For example, the engine control would not be affected by the heading angle, lateral speed, angular velocity and etc., when a ship far away from a wharf. When speed reduction is needed, a navigator just takes mainly the remaining distance to goal or present ship's speed into considerations. These facts are reflected well in newly designed neural network. For input units, 8 factors will be used which state the present ships conditions such as speed, heading angle, distance form goal point, etc. Among them funny thing are beam distance: $\mathrm{d} 1$ and $\mathrm{d} 2$. Fig. 3 explains the details. The $\mathrm{d} 1$ is beam distance to the imaginary line, which is used by navigators to help safe berthing. And the

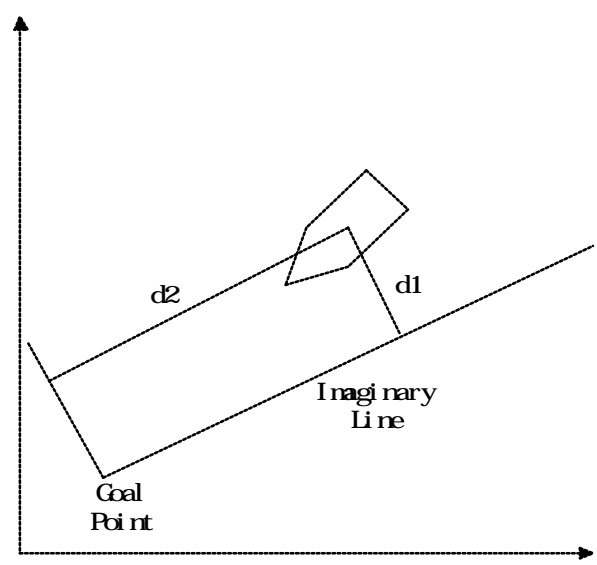

Fig.3 Coordinate system for berthing 


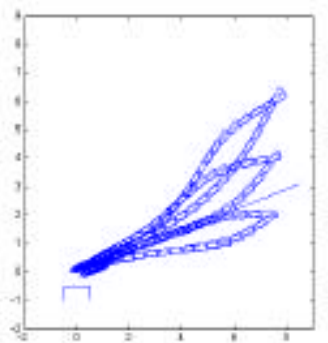

Fig. 4-1 training of 6 cases

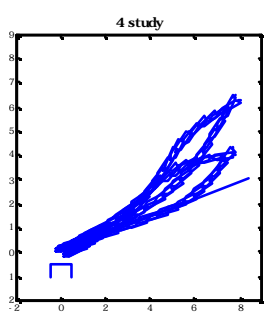

Fig.4-2 4 cases $\mathrm{d} 2$ is the rema ining distance to the goal point. These factors can explain the fact that navigators usually make imaginary line to goal point under berthing work. Even if $\xi, \eta$ can explain the location information, they are not enough to explain the ship's location information.

\section{PROCEDURE FOR LEARNING AND MAKING TEACHING DATA}

\subsection{Preparing for the teaching data}

This paper focuses mainly on how a parallel neural network will work effectively. So authors didn't try the automatic berthing problem using a tug or side thruster. This problem will be a next challenge. Automatic berthing mentioned here means that a ship stops near the goal point within $0.2 \mathrm{~m} / \mathrm{s}$ of speed and between 250-270deg of heading angle. Basically 6 types of teaching data were adopted as like the Fig. 4-1 where disturbance is not considered. 4 types of teaching data are also adopted to compare their effectiveness with 6 types of teaching data. Fig. 4-2 shows 4 types of teaching data.

\subsection{Procedure of training}
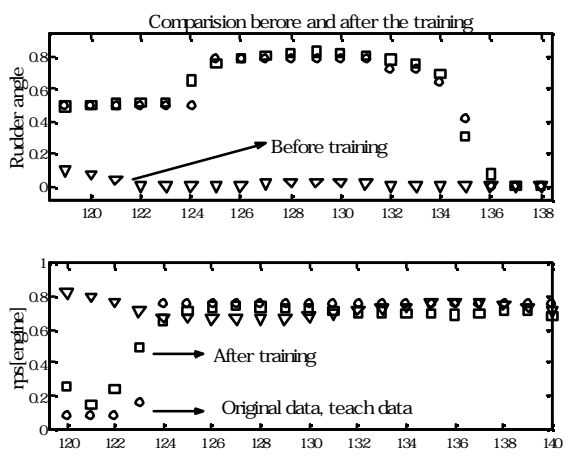

Fig. 5 Effect of training
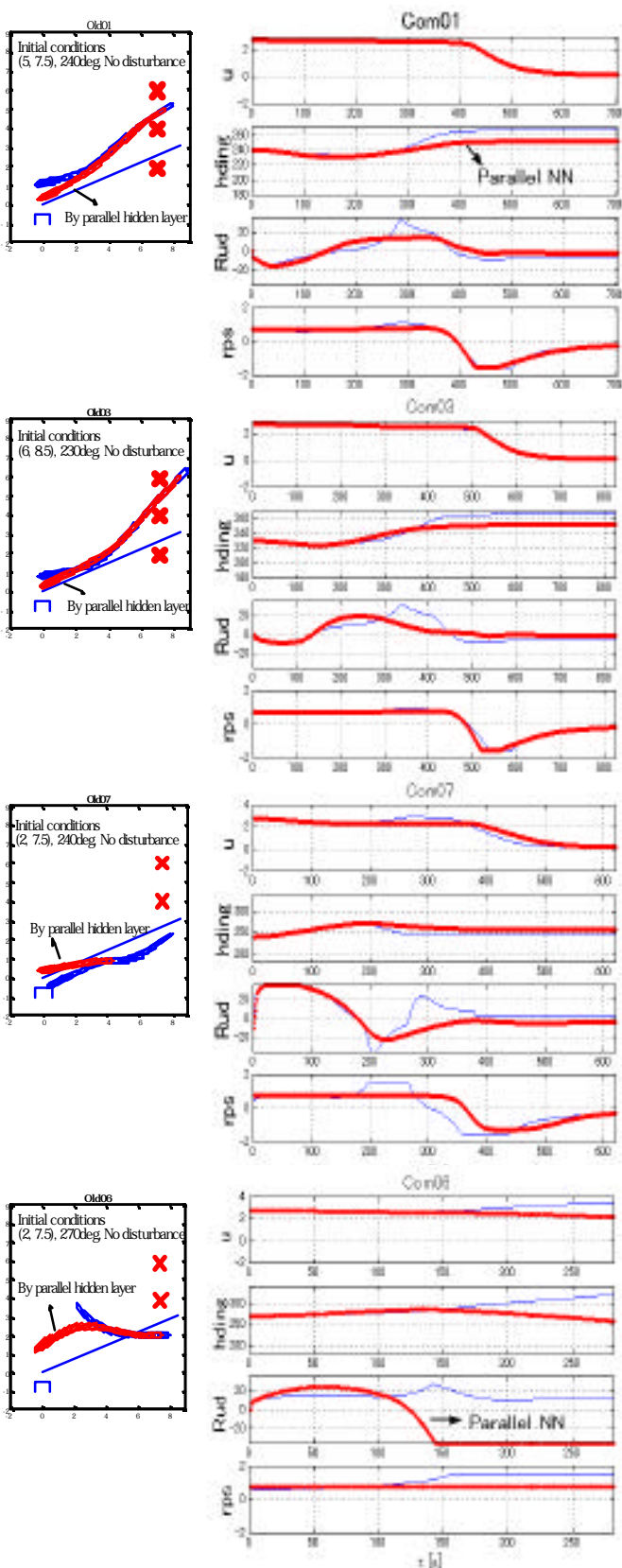

Fig. 6 Comparison of parallel and united NN when intial conditions are different with teaching data (cross marks indicate initial positions of teaching data)

Popular method of training, back propagation, is adopted. The Neural Network Toolbox from the MATLAB package has been used to train the model. Just variable learning rate method is used to minimize time taken. Generally learning rate is held constant throughout training. If the learning rate is set too high, the algorithm may oscillate and become unstable. If the learning rate is set too small, the algorithm will take too large time to converge. So variable learning rate is adopted here. Since a neural network in this paper have separated hidden layers, training is needed twice to make one set of weight and bias which will 

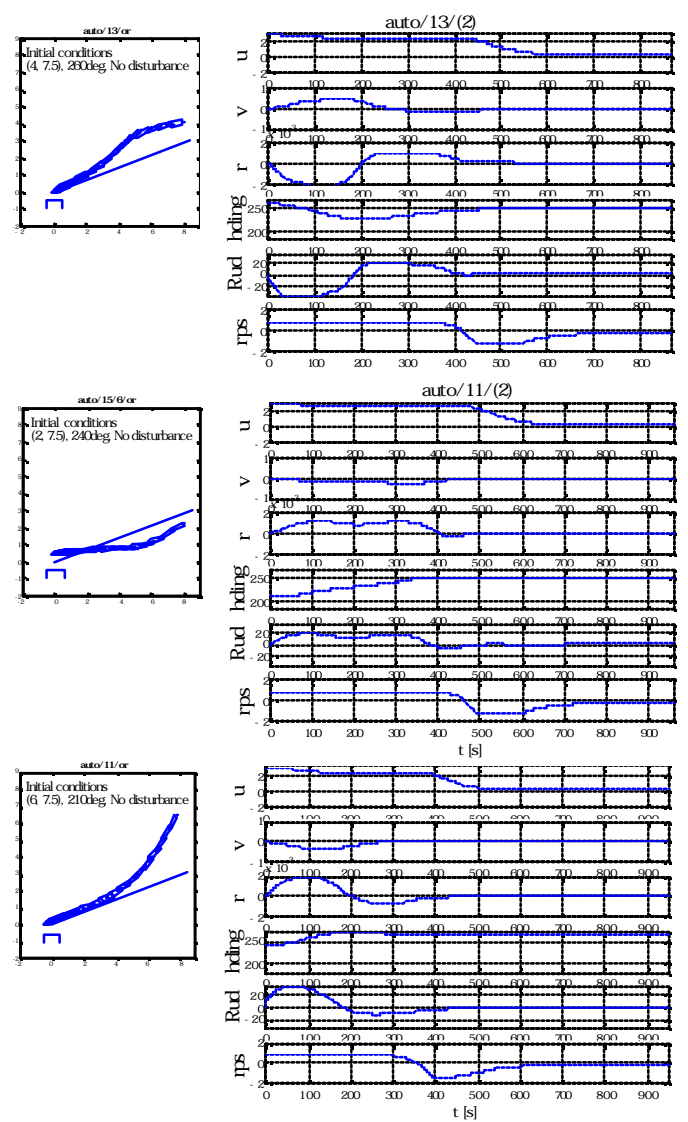

Fig. 7 Simulation results having same initial conditions with teaching data

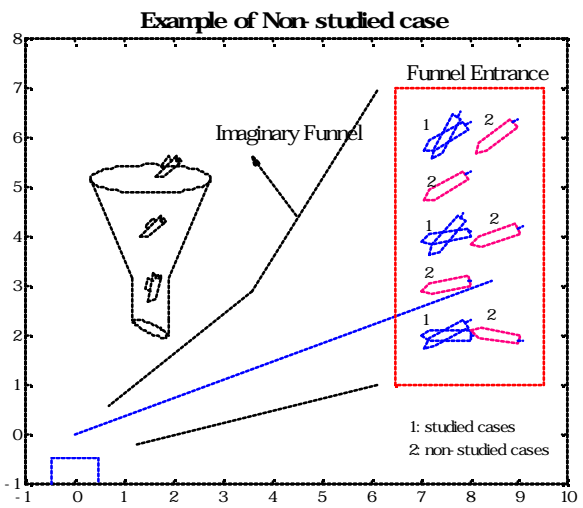

Fig. 8 Initial point of simulations

produce one set of output units. The group for rudder and the group for engine are trained separately. One example of these training is presented in Fig. 5. The figure explain very well the before and after training, where the circle shapes indicate the original data, teaching data, triangle means before training data and square means after training data respectively. It is easy to understand how much training is conducted well by this figure.

\section{SIMULATION RESULTS}

\subsection{Effect of parallel hidden layer control}

Fig. 6 shows comparisons with the parallel hidden layer and conventional layer. The red line (heavy line) is the results of separated control. The thin line is the results of united hidden layer. Cross marks indicate initial positions of the teaching data used for controller. This figure shows that even if the ship's states of two models are same in the initial stage, the output of the rudder and the engine are different because of the different hidden layer design. It is easy to understand that the parallel hidden layer has more stable and corrective control than the united hidden layer by these figures. Take a look at the last case of Fig. 6,even if both cases failed successful berthing, the parallel $\mathrm{NN}$ is showing its improvement in stopping ability and keeping track comparing with normal NN. Especially in the case of last two of Fig.6, even if weights and bias of Fig. 4-2 are used, in other words, starting positions and state are different with training data, but the parallel hidden layer's results is showing good ability comparing with that of united hidden layer.

\subsection{Simulations having the same initial conditions as training data}

Even if 6 cases were simulated, only 3 cases are presented as examples here. As it is shown in Fig. 7, the ability of stopping near the wharf and seeking a goal point is very good. Especially the stopping ability is good. It was possible to end within $0.2 \mathrm{~m} / \mathrm{s}$ at the wharf in all the cases. Also the final heading angles were within 250-270deg. The details are shown in Fig. 7.

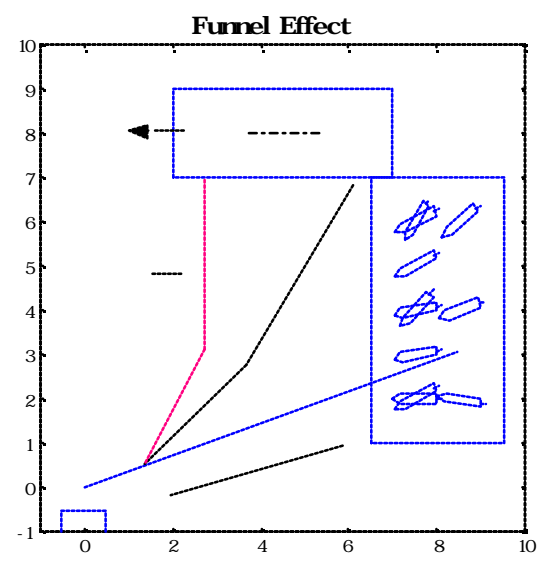

Fig. 9 Extension of funnel area 

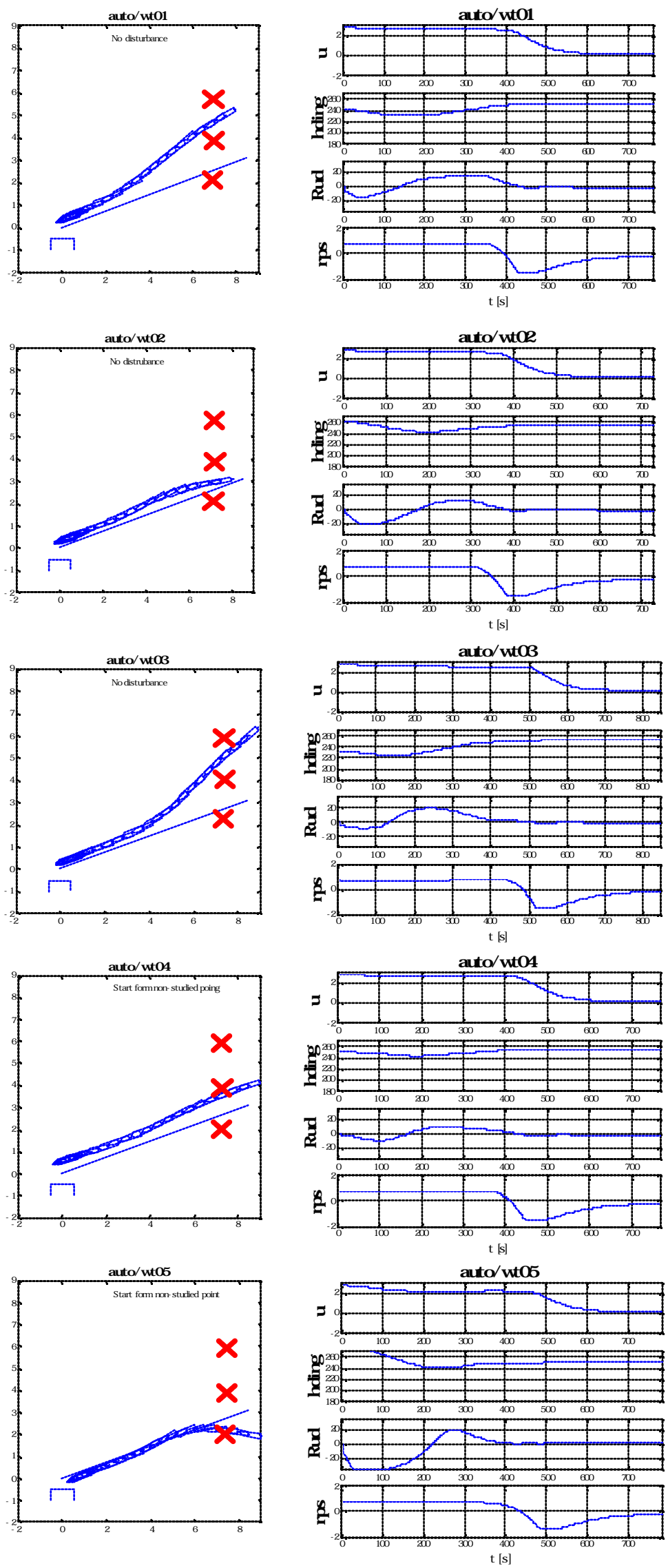

Fig. 10 Results having different initial conditions with teaching data (cross marks indicate initial positions of teaching data) 


\subsection{Results having different conditions with training data and Funnel Effect}

In this section, the funnel effect will be discussed. Many simulations that have different initial conditions with teaching data, are presented in this section. Fig. 8 shows the details. While the No. 1 represents simulation case where the same initial data as the teaching data are used, the No. 2 has different initial data with teaching data, but they carried good automatic berth. Figs. 10 shows the results where cross marks indicate initial positions of the teaching data. It is easy to understand with these figures that successful automatic berthing has been accomplished even if they have different initial conditions and different starting point with the teaching data. This is due to the interpolation ability of neural networks. ANN has great power of interpolation to solve a faced problem even if the situations are different with the teaching data. In Fig. 8 the square is marked. It is an area, which guarantees safe automatic berthing to the wharf from that area. The authors would like to call it the 'funnel area'. For example, when an object comes into the entrance of a funnel, the object should reach the opposite side of the funnel without escaping from the funnel passage like in Fig. 8. In this paper all simulations, which are done at the square area, are finished with good automatic berthing like Fig. 7 and 10. This funnel effect suggests the possibility that if more of these funnel areas are established like in Fig. 9, automatic berth can be realized from every direction and every dis tance.

\section{CONCLUSIONS AND DISCUSSIONS}

In this paper, a parallel ANN for ship berthing was discussed. In this paper, two groups of input units are considered to compose a parallel NN where hidden layers are split into two. The first group consider all of input units and anther group include only two input units such as remaining distance, d2, and a ship speed, u. But it can be said that additional research is needed to determine how input units should be separated. Conclusions of this paper can be drawn as follows

1) Newly designed ANN was used in automatic berthing problem as a controller.

2) A parallel ANN has good control ability comparing with normal ANN of united hidden layer.

3) The funnel area suggests the possibility that automatic berth from every direction can be accomplished.

4) Successful berthing has been accomplished even if under different initial condition and different starting point with teaching data

\section{REFERENCES}

Hiroko Itoh, "Berthing Control with Multi-Agent System", Journal of the Society of Naval Architects of Japan, Vol.184, Dec. 1998, p.639-648

H. Yamato, T. Koyama and T. Nakagawa, "Automatic Berthing using Expert System”, Proc. Of Workshop on Artificial Intelligence Control and Advanced Technology in Marine Automation(CAMS'92), p. 173-183, Geneva, Apr., 1992

H. Yamato and etc, "Automatic Berthing by the Neural Controller", Proc. Of Ninth Ship Control Systems Symposium, vol. 3, pp.3.183-201, Bethesda, U.S.A., Sep., 1990

K. Hasegawa, K. Kitera, "Mathematical Model of Maneuverability at Low Advance Speed and its Application to Berthing Control." Proc. Of The $2^{\text {nd }}$ Japan-Korea Joint Workshop of Ship and Marine Hydrodynamics”, pp.144-153, Osaka, June 1993.

K. Hasegawa, K. Kitera, "Automatic Berthing Control System Using Network and Knowledge-base", Journal of Kansai Society of Naval Architects of Japan, Vol. 220, Sept. 1993 p.135-143(in Japanese)

K. Kose etc.,"On a Computer Aided Maneuvering System in Harbors", Journal of the Society of Naval Architects of Japan, Vol.160, Dec. 1986, p.103-110

K. Kose etc. "On a Mathematical Model of Meuvering Motions of Ships in Low Speeds", JSNA of Japan, Vol. 155, June 1984, p 132-138

T. Koyama and Y. Jin, "A Systematic Study On Automatic Berthing Control( $1^{\text {st }}$ Report)", Journal of the Society of Naval Architects of Japan, Vol. 162, December 1987, p.201-210 (in Japanese) 